\title{
Timing Synchronization Method for Asymmetrically Clipped DC Biased Optical OFDM System
}

\author{
Yong Wang \\ College of Information \& Communication Engineering, Harbin Engineering University, Harbin, China \\ Email: wangyong@hrbeu.edu.cn
}

Received 19 April 2016; accepted 19 August 2016; published 25 August 2016

\begin{abstract}
In a typical intensity-modulated direct-detection optical system, the information is carried on the intensity of the optical signal, and therefore can only be positive. Existing synchronization methods for RF-based OFDM system cannot be used directly in asymmetrically clipped DC biased optical of DM (ADO-OFDM) system. In order to solve the above problem, according to the characteristics of ADO-OFDM signal, the modified training symbol is presented. The simulation results show that the modified synchronization method gives good performance.
\end{abstract}

\section{Keywords}

Optical Wireless Communication, ADO-OFDM, Timing Synchronization

\section{Introduction}

In recent years, interest in optical wireless communication as a promising complementary technology for radio frequency in short-rang communications has gained new momentum. Optical wireless communication offers attractive features, distinct from those of radio transmission, such as low cost, low power consumption, high security, high data rate, and so on. Besides, along with the continuing development of technology, optical wireless communication has been shown to be a good wireless interconnect technology for high capacity communication networks in the first and last mile. However, a large number of scatter exist in optical wireless communication link, and it will generate multiple scattering on the transmitted beam. The multiple scattering will make the received signal contain direct signal and multipath scattering signal. It is very easy to cause the receiver to generate serious inter-symbol interference. The quality of optical wireless communication is severely influenced. Recently, ACO-OFDM [1] has emerged as an effective solution to deal with the inter-symbol interference caused by multipath transmission. At low data rate, ACO-OFDM is more efficient in terms of optical power but it suffers from spectral inefficiency. In order to improve spectral efficiencies, a new technique called ADO-OFDM was proposed in [2]. This technique consists of transmitting simultaneously ACO-OFDM on the odd subcarriers and DCO-OFDM on the even subcarriers. However, one of the prominent problems of ADO-OFDM is timing synchronization, it restricts the system performance. Therefore, the new synchronization method will be studied to reduce the ADO-OFDM system timing synchronization error. 


\section{System Model}

Figure 1 shows the block diagram of the transmitter part of the ADO-OFDM system [2] [3]. The input data will be mapped to a complex vector $X=\left[X_{0} X_{1} \cdots X_{N-2} X_{N-1}\right]$. In the optical intensity- modulated direct-detection system, the baseband signals must be a real signal, not a complex signal. To obtain such a signal, the complex vector $X$ is constrained to have Hermitian symmetry. $X$ is divided into odd and even components, $X_{\text {odd }}$ and $X_{\text {even }} . X_{\text {odd }}$ and $X_{\text {even }}$ are input to separate IFFT blocks to produce $X_{\text {odd }}$ and $X_{\text {even }}$ respectively. The signal $X_{\text {odd }}$ at the IFFT output in the ACO-OFDM generation block is clipped at zero to produce ACO-OFDM signal, $x_{A C O}$. At the output of the IFFT block in the DCO-OFDM signal generation, a DC bias is added to the signal $x_{\text {even }}$ and any remaining negative peaks are clipped to give DCO-OFDM signal, $x_{D C O}$. Signal $x_{A C O}$ and $x_{D C O}$ are added together to give the signal $x^{\prime}$.

Figure 2 shows the receiver part of the ADO-OFDM system. There is a separate path to demodulate the ACO-OFDM symbols and another path to demodulate the DCO-OFDM symbols. The ACO-OFDM demodulation block is the same as that of the conventional ACO-OFDM receiver. However, to recover the symbols transmitted on the even subcarriers, an estimate of the ACO-OFDM signal is first generated. This is achieved by taking the odd subcarriers from $Y$ to obtain $Y_{\text {odd }}$. An estimate of the ACO-OFDM signal is calculated from $y_{\text {odd }}$ and subtracted from $y$ to recover the DCO-OFDM component.

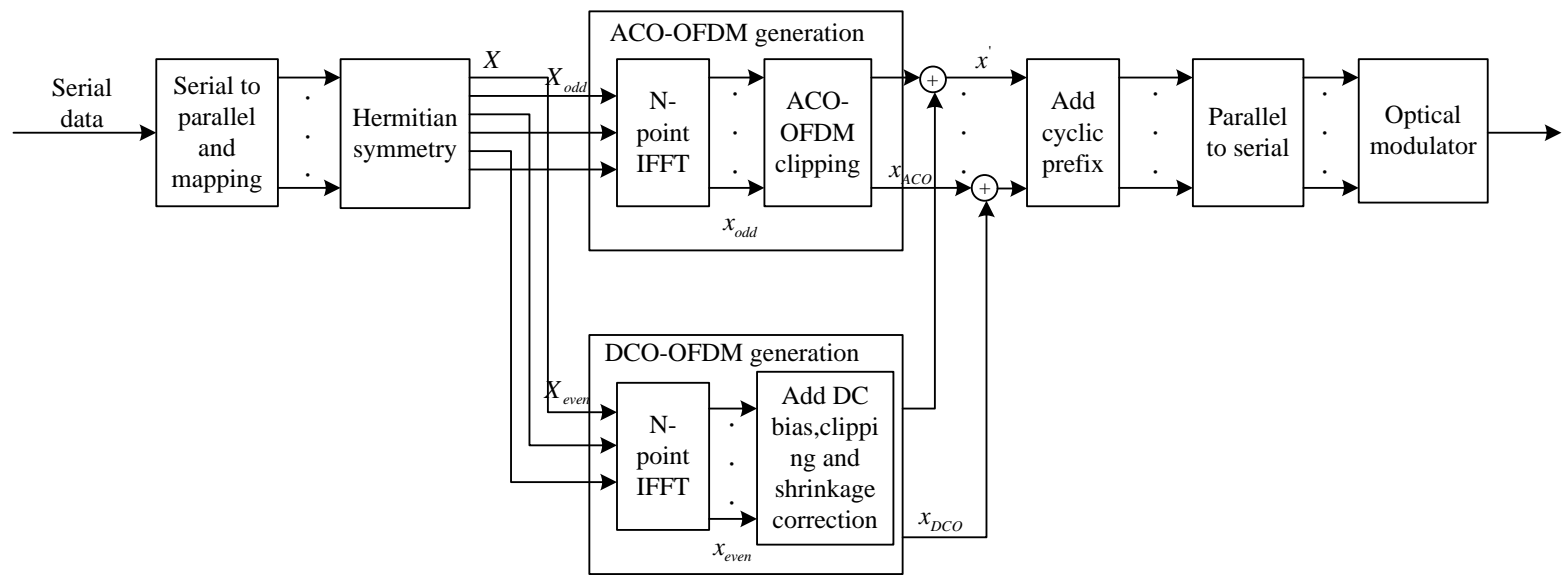

Figure 1. The transmitter of ADO-OFDM system.

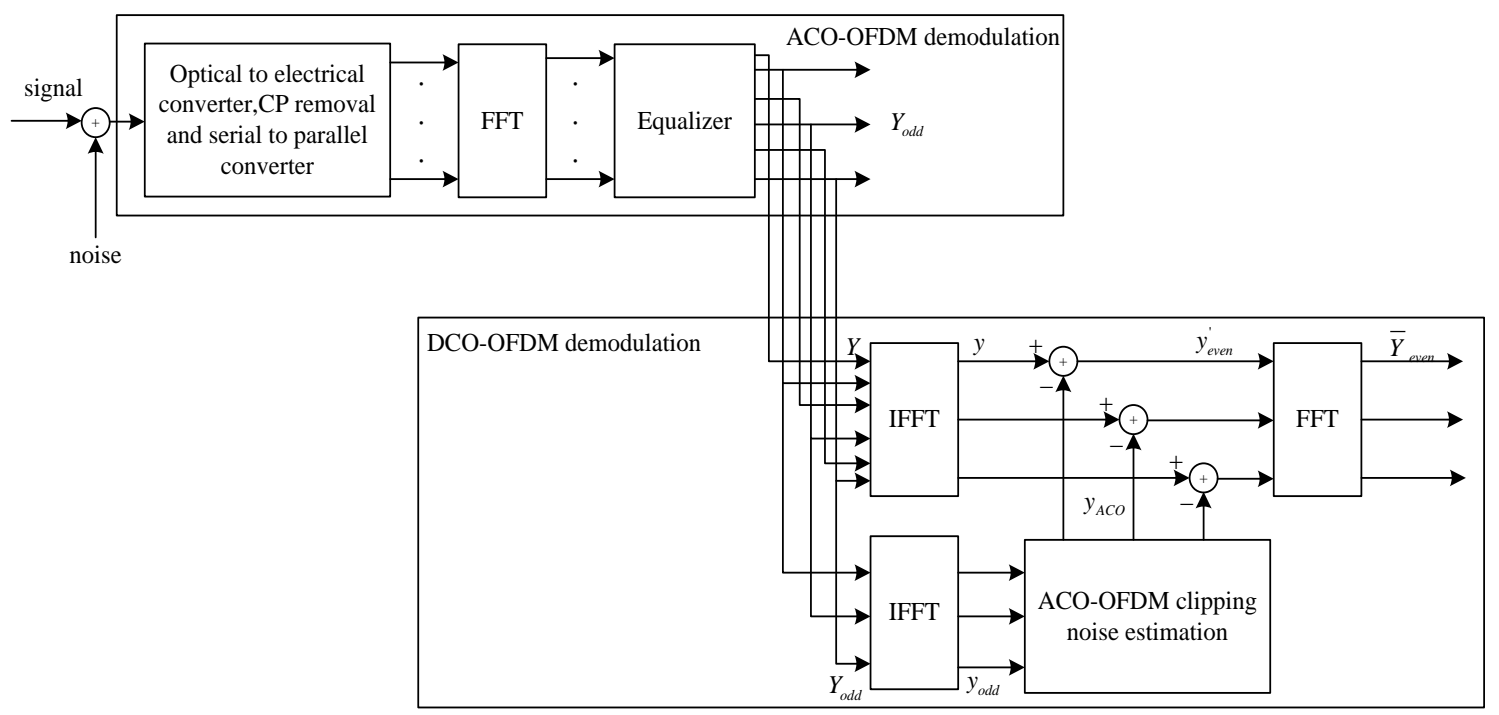

Figure 2. The receiver of ADO-OFDM system. 


\section{Timing Synchronization Methods}

\subsection{Schmidl's Method}

Schmidl's method [4] uses a symbol which consists of a repeated time domain sequence with the form $\left[A_{N / 2} A_{N / 2}\right]$ where $A_{N / 2}$ represents a sequence of length $N / 2$. The Schmidl's timing estimator finds the starting point of the symbol at the maximum point of the timing metric. The Schmidl's timing metric is given by

$$
M_{S c h}(d)=\frac{\left|P_{S c h}(d)\right|^{2}}{\left(R_{S h c}(d)\right)^{2}}
$$

where

$$
\begin{aligned}
& P_{S c h}(d)=\sum_{k=0}^{N / 2-1} r(d+k) r^{*}(d+N / 2+k) \\
& R_{S c h}(d)=\sum_{k=0}^{N / 2-1}|r(d+N / 2+k)|^{2}
\end{aligned}
$$

The timing metric has a plateau which leads to some uncertainty as to the start of OFDM symbol. The meansquare error of the Schmidl's estimator is quite large.

\subsection{Park's Method}

To reduce the uncertainty due to timing metric plateau and improve the timing offset eatimation, Park [5] proposed a time domain training symbol with the format $\left[B_{N / 4} C_{N / 4} B_{N / 4}^{*} C_{N / 4}^{*}\right]$, where $B_{N / 4}$ represents PN sequence of length $N / 4$, and $B_{N / 4}^{*}$ represents a conjugate of $B_{N / 4}$. To get impulse-shaped timing metric, $C_{N / 4}$ is designed to be the mirror image of $B_{N / 4}$. To make use of the property that $C_{N / 4}$ is symmetric with $B_{N / 4}$, a new timing metric can be written as

$$
M_{\text {Park }}(d)=\frac{\left|P_{\text {Park }}(d)\right|^{2}}{\left(R_{\text {Park }}(d)\right)^{2}}
$$

where

$$
\begin{aligned}
& P_{\text {Park }}(d)=\sum_{k=0}^{N / 2-1} r(d-k) r(d+k) \\
& R_{\text {Park }}(d)=\sum_{k=0}^{N / 2-1}|r(d+k)|^{2}
\end{aligned}
$$

\subsection{The Modified Timing Synchronization Method}

Because ADO-OFDM modulates the intensity of the light source, the signals must be real and positive. So time domain training sequences is constituted by real signals. Based on the above reason, a new training symbol is proposed in this paper. The time domain training symbol is defined as below:

$$
x_{\text {new }}=\left[\begin{array}{lll}
D_{N / 4} E_{N / 4} E_{N / 4}^{\text {mirror }} D_{N / 4}^{\text {mirror }}
\end{array}\right]
$$

where $D_{N / 4}$ and $E_{N / 4}$ are a sequence of real values, $D_{N / 4}^{\text {mirror }}$ is the mirror image of $D_{N / 4}$ and $E_{N / 4}^{\text {mirror }}$ is the mirror image of $E_{N / 4}$. The timing metric function is given by

$$
M(d)=\frac{|P(d)|^{2}}{(R(d))^{2}}
$$

where

$$
\begin{aligned}
& P(d)=\sum_{k=0}^{N / 2-1} r(d-k) r(d+k+1) \\
& R(d)=\sum_{k=0}^{N / 2-1}|r(d+k+1)|^{2}
\end{aligned}
$$




\section{Simulation Results and Analysis}

Schmidl and Park timing synchronization methods are all used in the RF-based OFDM system, and the training symbols is required to be bipolar and complex. Therefore, these methods cannot directly be applied to ADO-OFDM system. The data form of training symbols must be changed in order to apply the timing synchronization methods to optical wireless communication system. Figure $\mathbf{3}$ and Figure $\mathbf{4}$ shows the average timing metric of the adapted methods applied to ADO-OFDM system. Simulation parameters: $N=128$ subcarriers, a QPSK constellation on each of the available subcarriers, cyclic prefix length of $N / 8$, and 1000 random training symbols.

The timing metric curve still remains the plateau when the adapted Schmidl timing synchronization method is used. The adapted Park timing synchronization method has two extra peaks at $\pm N / 4$ because the training symbols are symmetry about the points. Therefore, it can be seen clearly that the adapted Schmidl and Park timing synchronization methods are not ideal for ADO-OFDM system. Figure 5 shows the average timing metrics of the modified timing synchronization method applied to ADO-OFDM system. Comparing with the adapted Park method, new timing synchronization method can effectively eliminates the two extra peaks at $\pm N / 4$. From the results of simulations, it is obvious that the performance of the modified timing synchronization method is better than other methods.

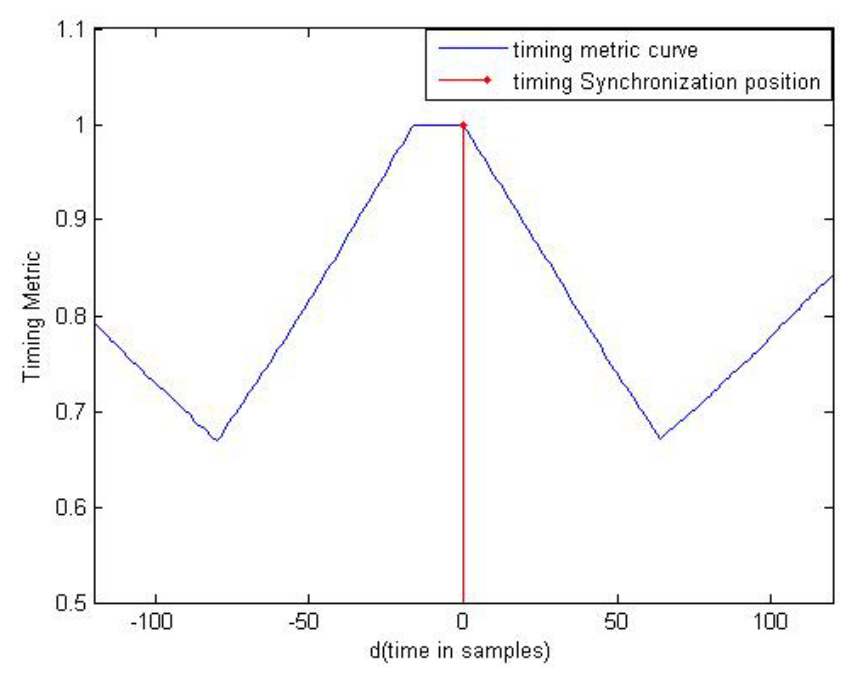

Figure 3. The Schmidl's timing metric in ADO-OFDM system.

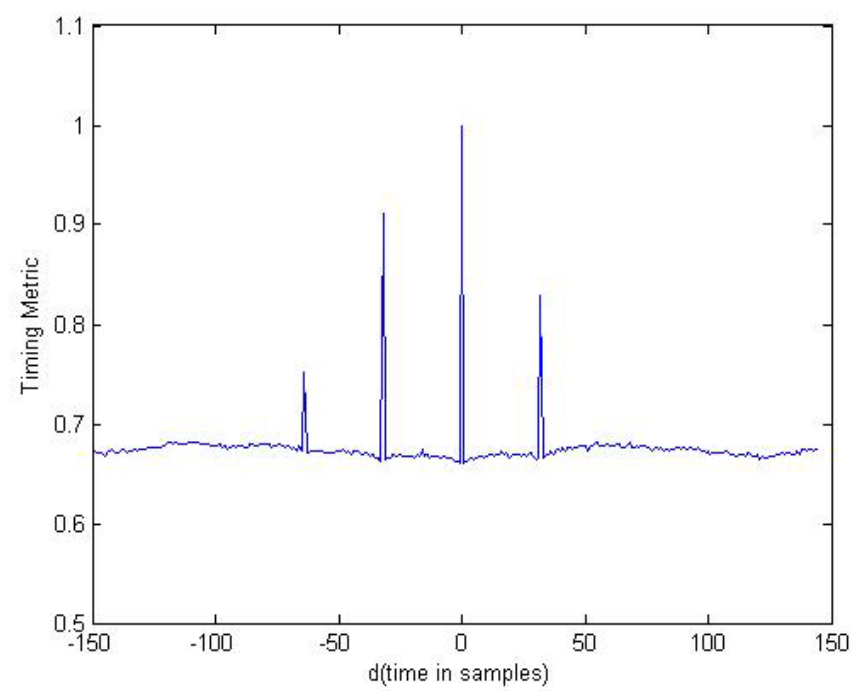

Figure 4. The Park's timing metric in ADO-OFDM system. 


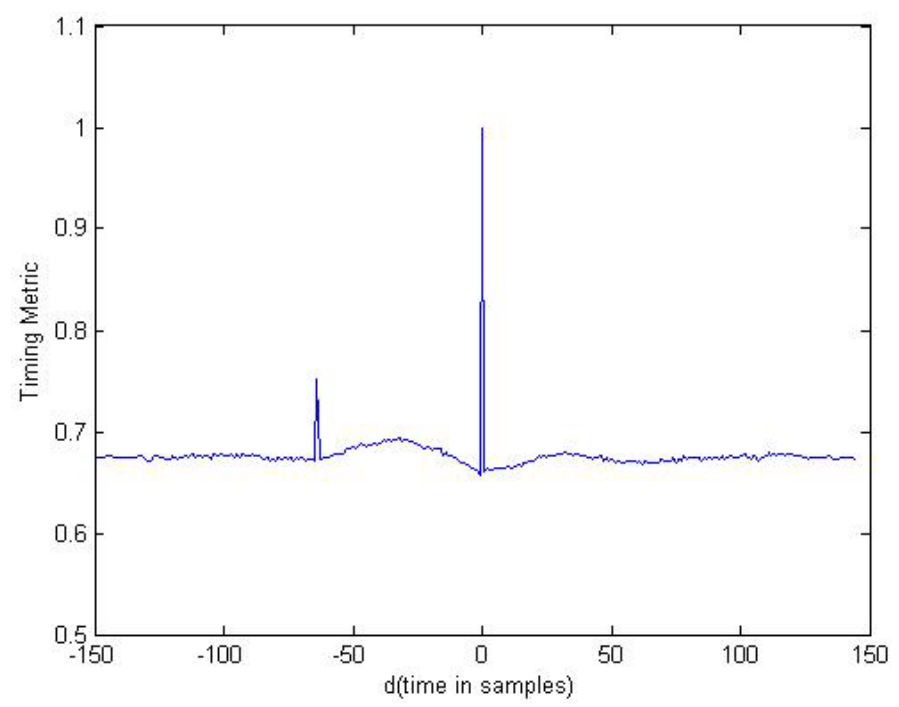

Figure 5. The modified timing synchronization metric in ADO-OFDM system.

\section{Conclusion}

The conventional timing synchronization methods for RF-based OFDM system are studied. The timing synchronization methods are adapted and applied to ADO-OFDM system. The analysis shows that these methods do not perform well in ADO-OFDM system. Therefore, a modified training symbol is proposed in this paper. The simulation results show the modified timing synchronization method can obtain higher timing accuracy.

\section{Acknowledgements}

This work was financially supported by the Natural Science Foundation of China (61275082).

\section{References}

[1] Armstrong, J and Lowery, A.J. (2006) Power Efficient Optical OFDM. Electron. Lett, 42, 370-372. http://dx.doi.org/10.1049/el:20063636

[2] Dissanayake, S.D., Panta, K. and Armstrong, J. (2011) A Novel Technique to Simultaneously Transmit ACO-OFDM and DCO-OFDM in IM/DD Systems. IEEE GLOBECOM Workshops, 782-786.

http://dx.doi.org/10.1109/glocomw.2011.6162561

[3] Dissanayake, S.D. and Armstrong, J. (2013) Comparison of ACO-OFDM, DCO-OFDM and ADO-OFDM in IM/DD Systems. Journal of Lightwave Technology, 31, 1063-1072. http://dx.doi.org/10.1109/JLT.2013.2241731

[4] Schmidl, T.M. and Cox, D.C. (1997) Robust Frequency and Timing Synchronization for OFDM. IEEE Transactions on Communications, 45, 1613-1621. http://dx.doi.org/10.1109/26.650240

[5] Park, B., Cheon, H., Kang, C. and Hong, D. (2003) A Novel Timing Estimation Method for OFDM Systems. IEEE Communications Letters, 7, 239-241. http://dx.doi.org/10.1109/LCOMM.2003.812181 


\section{Submit or recommend next manuscript to SCIRP and we will provide best service for you:}

Accepting pre-submission inquiries through Email, Facebook, LinkedIn, Twitter, etc.

A wide selection of journals (inclusive of 9 subjects, more than 200 journals)

Providing 24-hour high-quality service

User-friendly online submission system

Fair and swift peer-review system

Efficient typesetting and proofreading procedure

Display of the result of downloads and visits, as well as the number of cited articles

Maximum dissemination of your research work

Submit your manuscript at: http://papersubmission.scirp.org/ 